\title{
Correction to: Consensus on international guidelines for management of groin hernias
}

\author{
Nadine van Veenendaal ${ }^{1} \cdot$ Maarten Simons $^{2}$ - William Hope ${ }^{3}$. Sathien Tumtavitikul ${ }^{4}$. Jaap Bonjer ${ }^{1}$ on behalf of the \\ HerniaSurge Group
}

Published online: 22 April 2020

(c) Springer Science+Business Media, LLC, part of Springer Nature 2020

\section{Correction to: Surgical Endoscopy} https://doi.org/10.1007/s00464-020-07516-5

In the Acknowledgments, Lars N. Jorgensen of the HerniaSurge Group was incorrectly listed as:

"Lars Jorgensen, Bispebjerg Hospital, University of Copenhagen, Copenhagen, Denmark."

The correct listing should have read:

"Lars N. Jorgensen, Bispebjerg Hospital, University of Copenhagen, Copenhagen, Denmark."

The members of the HerniaSurge Group were listed in Acknowledgements section.
Publisher's Note Springer Nature remains neutral with regard to jurisdictional claims in published maps and institutional affiliations.

The members of the HerniaSurge group are listed in Acknowledgements section.

The original article can be found online at https://doi.org/10.1007/ s00464-020-07516-5.

Maarten Simons

m.p.simons@olvg.nl

1 Department of Surgery, Amsterdam University Medical Center, Amsterdam, The Netherlands

2 Department of Surgery, OLVG Hospital, Oosterpark 9, 1091 AC Amsterdam, The Netherlands

3 Department of Surgery, New Hanover Regional Medical Center, Wilmington, USA

4 Department of Surgery, Vichaiyut Hospital, Bangkok, Thailand 